\title{
Skills, determinants and attitudes towards leadership among undergraduates in medicine
}

\author{
Dissanayake, D.W.N. ${ }^{1}$, Gunasekara, D.P.A.V. ${ }^{1}$, Nanayakkara, U. ${ }^{1}$, Majeed, $S .{ }^{1}$, \\ Dissanayake, D.D.M. ${ }^{2}$
}

\begin{abstract}
Background: Good leadership is fundamental for providing a better healthcare. The objective of this study was to evaluate the leadership skills, determinants and attitudes in medical undergraduates.

Methods: Self and Rater versions of the Authentic Leadership Questionnaire (ALQ) and a formulated questionnaire based on Medical Leadership Competency Framework (MLCF) were distributed among first year students who were followed up in the final year with the same assessments. Skills were evaluated with an average ALQ score, under four domains; transparency (TR), ethical skills (ES), balanced processing (BP) and self-awareness (SA). Determinants of leadership skills were assessed by a self-administered questionnaire.
\end{abstract}

Results: Students in the first year $(n=115$; females $=64$, males $=51)$, had lower leadership skills score (2.74 \pm 0.38 ; mean $\pm S D)$ than in the final year $(n=101$; females $=52$, males $=49 ; 3.05 \pm 0.43 p<0.001)$. Highest mean score in the first year was for SA $(2.85 \pm 0.55)$ and the lowest was for ES $(2.48 \pm 0.47)$. Significant improvement in all the ALQ domains were observed in the final year than the first year $(p<0.05)$. Males had a higher mean ALQ score in the first year and a significantly higher score $(3.14 \pm 0.47)$ than females $(2.97 \pm 0.37 ; p=0.04)$ in the final year. Knowledge on medical leadership was good in the first $(4.39 \pm 0.33)$ and the final year $(4.38 \pm 0.69 ; p=0.85)$. Students who had done extracurricular activities had good leadership skills $(3.35 \pm 0.49)$.

Conclusions: Students had improved leadership skills in the final year than in the first year. Males had better leadership skills and significant improvement than females. Including leadership training in medical curricular is recommended.

Keywords: Leadership, undergraduates, medicine, skills, attitudes, determinants

\section{Introduction}

Leadership is the active and productive contribution of individuals within their working roles to maintain and enhance performance of an organization (Spurgeon et al. 2008;_Saravo et al. 2017).

${ }^{1}$ Department of Physiology, Faculty of Medicine, University of Colombo

${ }^{2}$ Princess Alexandra Hospital, Hamstel Road, Harlow, Essex, CM20 1QX, UK

Corresponding author: Dilshani WN Dissanayake dilshanid@physiol.cmb.ac.lk

DOI: http://doi.org/10.4038/seajme.v15i2.368
A clinician has varied leadership roles in his/her daily works; including formulating and finalizing care plans, teaching trainees, handling multiprofessional teams etc (Saravo et al. 2017). Thus, good medical leadership is vital for delivering high-quality healthcare. However, medical career progression has traditionally been seen to lack leadership qualities (Gulati et al. 2021; Spurgeon et al. 2008; Saravo et al. 2017; Kiesewetter et al. 2013; Steinert et al. 2012). Individual standards of health care professionals, with variations in their quality of medical care, have occasionally resulted in catastrophic lapses in the standards of care

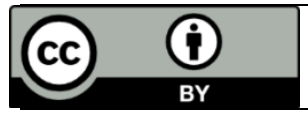

(C) SEAJME. This is an Open Access article distributed under the terms of the Creative Commons Attribution License (http://creativecommons.org/licenses/by/4.0/), which permits unrestricted use, distribution, and reproduction in any medium, provided the original author and source are credited 
provided (Warren \& Carnall 2011). Hence, it's essential for the doctors to develop strong leadership skills.

Until recently, leadership training was not included or properly assessed globally both during undergraduate and post-graduate periods through an approved time-tested curricular [Spurgeon et al. 2008; Saravo et al. 2017; Kiesewetter et al. 2013; Steinert et al. 2012; Baker \& Denis 2011; Abbas et al. 2011). Recent increase in evidence, linking clinical leadership to performance of units, has led to more focus on leadership development for doctors, starting as early as during the undergraduate period and continuing throughout their careers. In UK, leadership skills are mandatory for all doctors as laid out in the General Medical Council (GMC) publication Good Medical Practice and has been incorporated into both the undergraduate and post-graduate curricula as well [NHS Institute for Innovation and Improvement 2010; Leadership and management for all doctors 2012). The Medical Leadership Competency Framework (MLCF) developed by the Academy of Medical Royal Colleges and NHS Institute for innovation and improvement outlines the leadership competencies emphasizing that doctors need to become more actively involved in the planning, delivery and transformation of health care services.

Different assessment strategies have been used in industries to assess leadership skills (Kang \& Jin 2015). One such methodology is the assessment of authentic leadership skills which are applicable to any set up and not affected by culture, economy and other confounders (Walumbwa et al. 2008). The main domains of this are the self-awareness, transparency, ethical/moral values and balanced processing. Recent studies have highlighted the importance of integrating leadership training and provision of actual clinical training opportunities to practice these skills at undergraduate level (Goldstein et al. 2009) and have evaluated the potential methods of training medical graduates for community leadership (Saravo et al. 2017; Kiesewetter et al. 2013; Goldstein et al. 2009). Training methodologies such as mentoring, coaching, simulation-based learning with roleplay followed by active learning in clinical setting and networking have been proposed (Spurgeon et al. 2008; Saravo et al. 2017; Kiesewetter et al. 2013; Steinert et al. 2012; Baker \& Denis 2011; Abbas et al. 2011). Such methodologies will help to change currently held negative attitudes and beliefs. Furthermore, provision of good role models is a mandatory requirement to create future leaders (Warren \& Carnall 2011; Baker \& Denis 2011; Abbas et al. 2011).

It's important to evaluate leadership skills in medical students at a very early stage in their carrier and to take measures to enhance these skills with continuous assessment and monitoring. Thus, the objective of this study was to evaluate the skills, determinants and attitudes towards leadership in medical undergraduates in their first and the final year and its findings provide groundwork for curricular development aiming to improve leadership skills in the medical profession and to facilitate and promote further studies on medical leadership.

\section{Methods}

The current study was carried out in the Faculty of Medicine, University of Colombo as a follow up study. The first phase involved the first-year medical students and the second phase was a follow up study of the first-year graduates who were re-assessed in their final year after five years of study. The students in the first year were assessed in their second semester and in the final year they were assessed in the midyear, during their third professorial appointment. These points of data collection were chosen considering provision of adequate time for the first-year students to adjust to the new environment and for final years having adequate time before their final academic examination. It was also dependent on the approval given by the administration, convenience and time available for the students.

All the consenting students in the relevant batches were recruited to the study. Selfadministered pre and pilot tested 
questionnaires (as described below) were distributed among the students at the end of a lecture during a pre-arranged time slot, after providing guidance and obtaining informed written consent. Investigators were available to clarify any issues. A box was kept to submit the completed questionnaires.

Background information was obtained via a questionnaire to evaluate the determinants; age, gender, home town, religion, school, extracurricular activities done at school and the faculty, number of advanced level (A/L) examination attempts, $A / L$ results, monthly family income and courses/ workshops followed in leadership. The students were expected to indicate as to how they perceive themselves as leaders in a rating scale from poor to excellent and also whether they require training in leadership skills.

Self and rater survey of the Authentic Leadership Questionnaire tested and validated by several studies was used to assess leadership skills (ALQ; version 1.0, 2007) (Walumbwa et al. 2008). Permission was obtained from the authors to use the tool. The ALQs are designed to measure the components of authentic leadership using scenarios/questions with related responses (Walumbwa et al. 2008). Scoring is done based on the marks allocated for each response (Walumbwa et al. 2008). These responses are designed in a way that the respondents cannot easily predict the scores allocated for each response (Walumbwa et al. 2008). A referenced and formulated Questionnaire based on Medical Leadership Competency Framework (MLCF) was utilized to assess knowledge.

Leadership skills were evaluated based on the average score for the ALQ as per standard guidance given with the tool (each of the 16 items rated from 1-4); those with an average score of $<3$ were classified as poorly skilled and $\geq 3$ as having good leadership skills. The 16 items were assessed under four main categories, transparency (TR), ethical skills (ES), balanced processing (BP) and selfawareness (SA) of ALQ. The rater version evaluated the participants' assessment of their immediate leader which included the same items as in the self-ALQ and items were rated and scoring were done in the same manner. The immediate leaders were the group monitors, batch representatives and union leaders. Students were allowed to select a "student leader" out of the above and answer the questions based on the behaviors that they have observed in the leader.

Knowledge and attitudes were assessed using MLCF, which contained five components; personal qualities, working with others, managing services, improving services and setting directions. The questionnaire consisted of 30 statements and each statement was given a score from a scale of $1-5$. An average score of 4 or more for each component and also for the averaged total score was considered as having good knowledge.

Data analysis was done using descriptive and comparative analytical tools in SPSS v21. Percentages and mean values were used for descriptive analysis and unpaired student $T$ test was used to compare score differences in relation to possible determinants e.g., gender, "leaders". One way ANOVA was used to assess differences in scores in the first and final year.

\section{Results}

164 out of the 180 first year medical students responded and only those who had fully completed all the questionnaires were selected for the current analysis. In the first-year students ( $n=115$; males- 51 , females- 64 ; age, $21.5 \pm 0.4$ years; mean $\pm S D$ ), the mean score for the total ALQ assessment was below 3 (2.74 \pm 0.38$)$. Among this group $41.7 \% \quad(n=48)$ had a total score indicating good leadership skills $(3.25 \pm 0.18)$ while $58.3 \%$ had scores indicating poor leadership skills $(2.59 \pm 0.32$; Figure.1). Out of the ALQ components, selfawareness had the highest score obtained by the whole group $(n=115 ; \quad S A=2.85 \pm 0.55)$ followed by balanced processing $(\mathrm{BP}=2.84 \pm 0.61) \quad$ and transparency $(T R=2.79 \pm 0.44)$. Ethical skills had the lowest score (ES=2.48 \pm 0.47 ; Figure.2). 

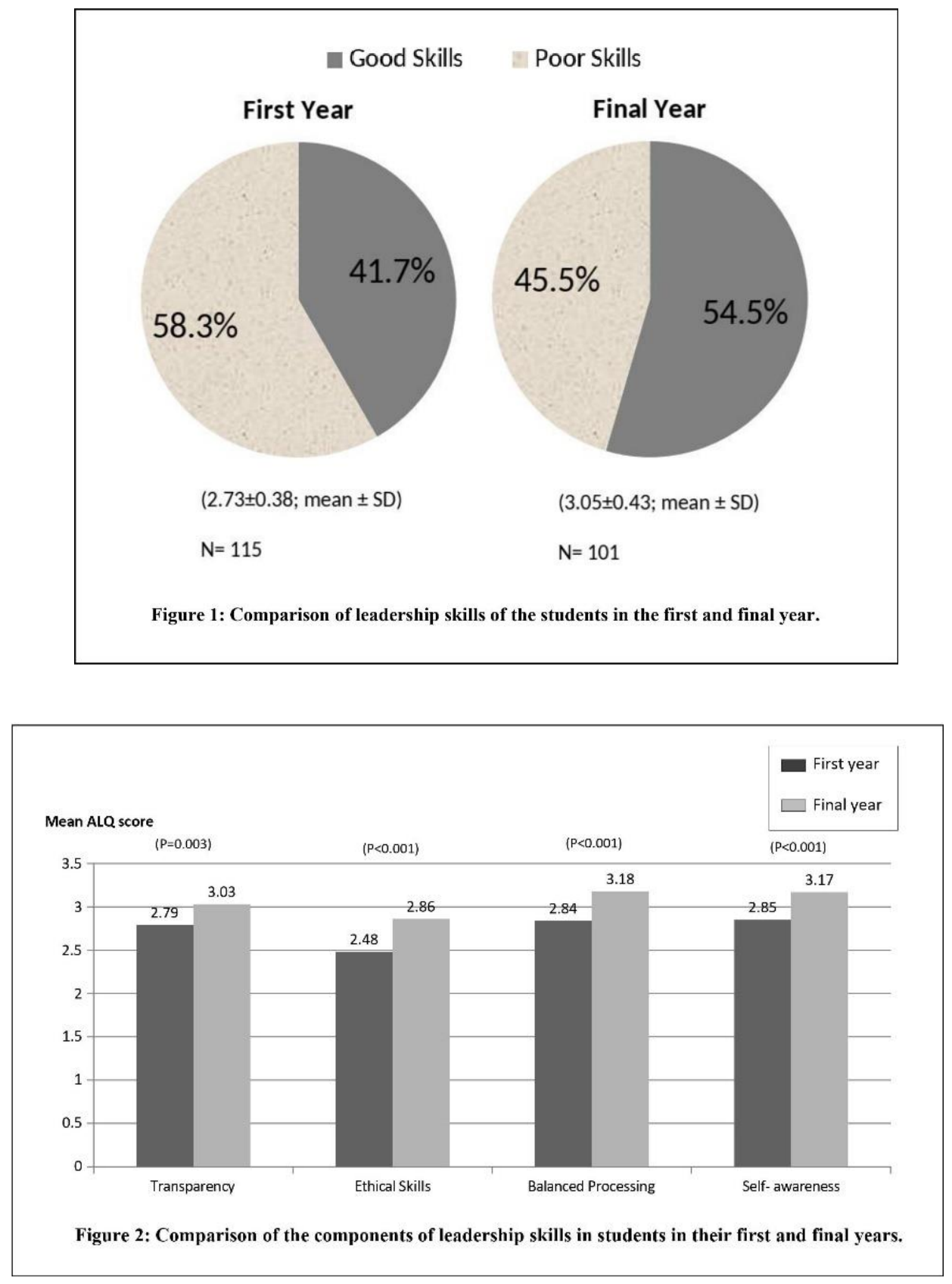

Less than $50 \%$ of females (40.6\%) and males (43.1\%) obtained total scores for good leadership skills. Females had poor total leadership score $(2.68 \pm 0.36)$ than males
(2.82 $\pm 0.46 ; p=0.086)$ in all the components out of which the ethical skills were significantly lower in females $(2.36 \pm 0.52)$ than males $(2.71$ $\pm 0.46 ; p=0.01$; Figure 3 ).

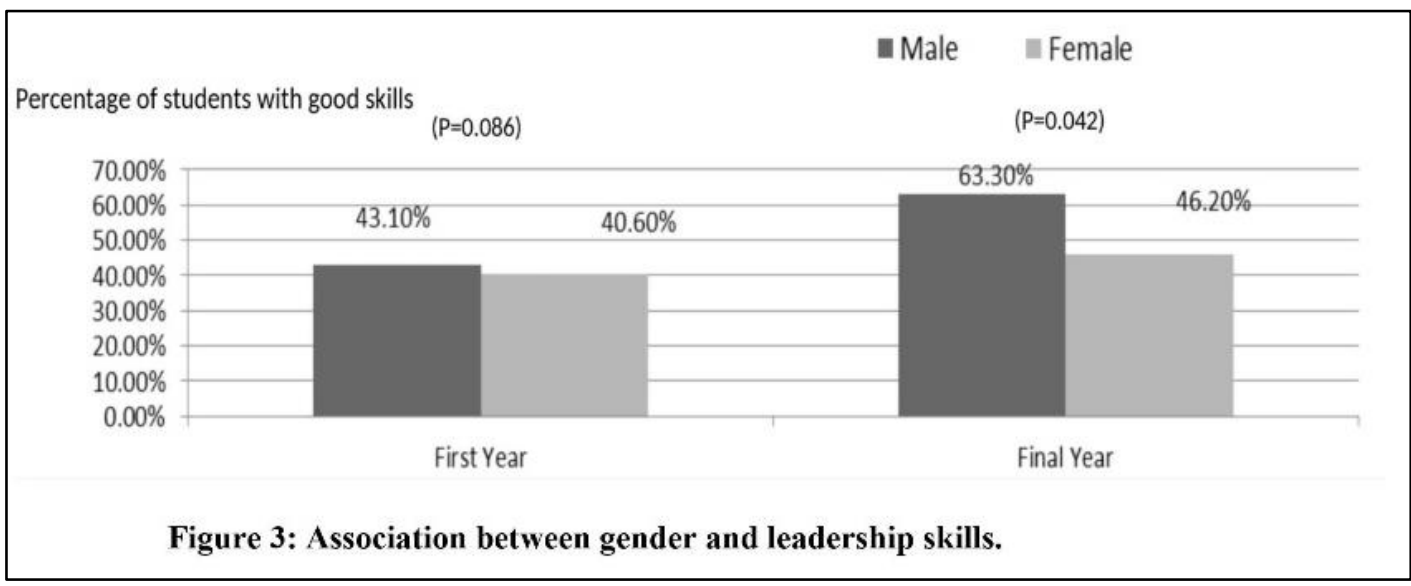


The students who entered through the first attempt at the entry examination (advanced level=A/L) had a higher total score $(60.3 \%$, $\mathrm{n}=69,2.80 \pm 0.45)$ than those who entered via the $2^{\text {nd }}$ and third attempt $(39.7 \%, n=46$, $2.67 \pm 0.35 ; \mathrm{p}=0.11)$. The students with 3 distinction passes for all the subjects at $A / L$ (i.e.,3As; $56.3 \%$ ) had a higher total score (3.29 \pm 0.29 ) than the students without 3 As $(2.28 \pm 0.47, p=0.05)$. The balance processing skills were significantly higher in students with $3 A$ passes $(3.03 \pm 0.34)$ than others $(2.25 \pm 0.29$; $\mathrm{p}=0.01$ ). There was no relationship between family income, type of school and the area of residence with their scores for leadership skills.

In the first years the "student leaders" had a higher total score than the participants $(2.91 \pm$ 0.53; $\mathrm{p}<0.001$; Figure 4) with significantly higher scores in all the aspects of leadership skills compared to the scores of the participants ( $\mathrm{TR}=3.31 \pm 0.44, \mathrm{ES}=2.94 \pm 0.56, \mathrm{BP}=3.17 \pm 0.05$, $S A=3.20 \pm 0.56 ; p<0.01)$. The knowledge on different aspects of medical leadership was good in the whole group $(4.39 \pm 0.33)$ and was higher in the group with good leadership skills $(n=67 ; 4.66 \pm 0.43)$ than those with poor skills $(n=48 ; 4.34 \pm 0.54 ; p=0.16)$ in all the areas. In the group with poor leadership skills, the mean values for personal qualities $(4.30 \pm 0.46)$, working with others $(4.35 \pm 0.53)$, managing services $(4.30 \pm 0.55)$, improving services (mean $4.03 \pm 0.59)$, and setting directions $(4.30 \pm 0.58)$ were lower than personal qualities $(4.65 \pm 0.31)$, working with others $(4.68 \pm 0.31)$, managing services $(4.60 \pm 0.37)$, improving services $(4.65 \pm$ $0.43)$, and setting directions $(4.58 \pm 0.43)$ of the group with the good leadership skills. However, the differences were not statistically significant.

In response to the question on the perception on leadership skills, $85.4 \%$ of the students rated themselves as good or average leaders with $14.6 \%$ rating themselves as very good leaders. None considered themselves to be excellent or poor leaders. Majority (82.9\%) indicated that they would benefit from formalised leadership training programmes.

When the students were re-assessed in the final year, their mean age was $24.94 \pm 0.86$ years. 132 out of 150 students responded. Out of the students who fully completed ALQ $(n=101$; females $=52$, males $=49)$, the total leadership skills score was better compared to their first year $(3.05 \pm 0.43 ; p<0.001$, Figure 1) with higher percentage of students with good leadership skills $(54.5 \%, n=55)$. Final year students had improved in all the components of ALQ than the first year; TR- $(3.03 \pm 0.54$; $2.79 \pm 0.44 ; \quad p=0.003), \quad E S-(2.86 \pm 0.64$; $2.48 \pm 0.47 ; \quad \quad p<0.001), \quad$ BP $-(3.18 \pm 0.56$; $2.84 \pm 0.61 ; p<0.001) S A-(3.17 \pm 0.57 ; 2.85 \pm 0.55$; $\mathrm{p}<0.001$; Figure2.).

In the final year $46.2 \%$ of females and $63.3 \%$ of males had scores indicating good leadership skills. Both females and males had significant improvements in the scores for leadership skills in the final year than the first year (females $2.68 \pm 0.36, \quad 2.97 \pm 0.37, \quad \mathrm{p}=0.04 ;$ males, $2.82 \pm 0.46,3.14 \pm 0.47, p<0.01)$. Improvement in all the leadership competencies in males was significantly higher than those of females, creating a significant difference in skills between gender in the final year than the first year (females-2.97 \pm 0.37 , males-3.14 \pm 0.47 ; $\mathrm{p}=0.042$; Figure 3 ). Ethical skills were still lower in females compared to males $(2.91 \pm 0.62$, $2.62 \pm 056 ; p=0.226$ ), though it was not statistically significant.

In the final year, the "student leaders" had lower scores than the participants $(3.05 \pm 0.43$, "leader"-2.75 $\pm 0.68 ; p<0.001$; Figure4). There was no significant difference in knowledge between first years $(n=115 ; 4.39 \pm 0.33)$ and final years $(n=111 ; 4.38 \pm 0.69 ; p=0.85)$. The group with good leadership skills had a significantly better knowledge score $(n=55$, $4.62 \pm 0.51$ ) than the group with the poor leadership skills $(n=46 ; 4.21 \pm 79 ; p=0.01)$. There was an association between participation in sports and extracurricular activities with good leadership skills. Students who have participated in both sports and non-sport related extracurricular activities had the highest leadership scores of the group.

Students who did not do extracurricular activities showed poor balanced processing skills $(3.12 \pm 0.40)$ than those who did both sports and none sports type of extracurricular activities (3.35 $\pm 0.49 ; p=0.032)$. 


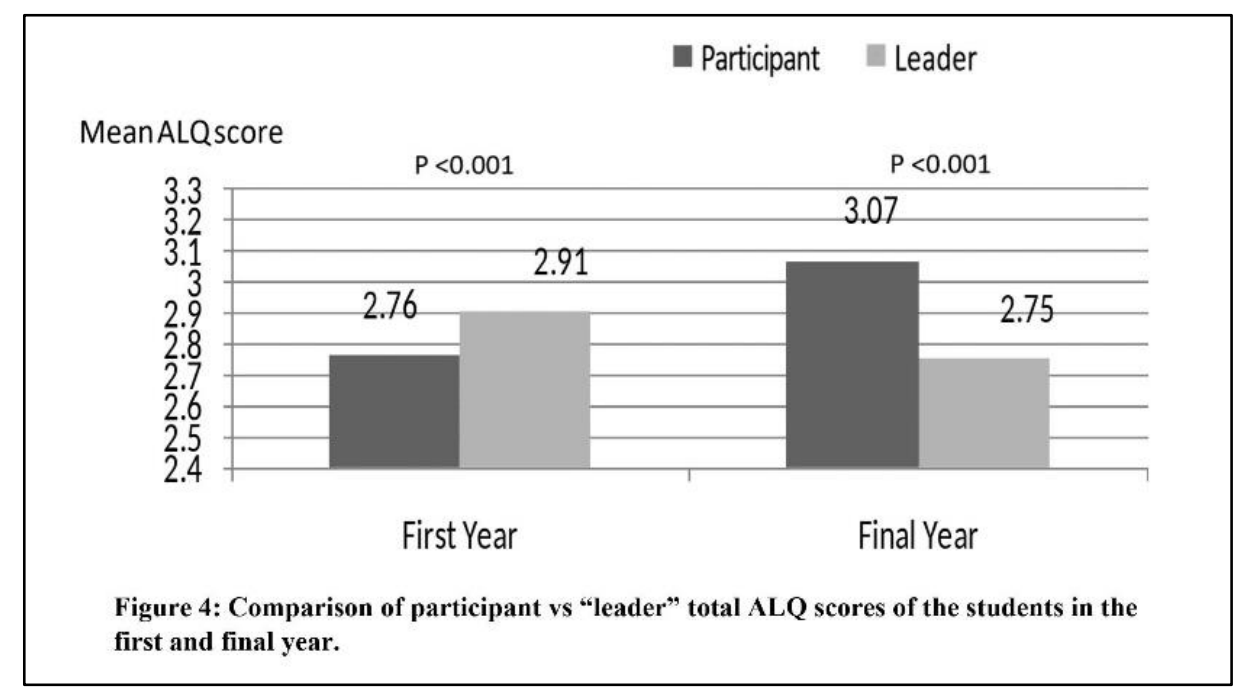

Students who participated in extracurricular activities from school and continued them in the faculty had good ALQ scores compared to the students who did sports only at school or only at the faculty. There was no association between marks of end of stream and continuous assessments of the faculty with the student's leadership skills $(p=0.597)$.

\section{Discussion}

The findings of the current study demonstrate low leadership skills in all the ALQ domains with significant deficiency in ethical skills of the firstyear students and the lower skills in females compared to males. Though leadership skills of females in medicine is not thoroughly researched, some studies suggest that females are better transformational leaders with higher effectiveness compared to males (Eagly et al. 2003). It is well known, but not openly expressed, that females are stereotyped to be inferior to men for leadership roles in the world. In UK, though almost half of the doctors are women, higher strata in medicine have inordinately a lesser number of females and thus the Royal College of Physicians have considered motivating women to take up more leadership roles as one of their key goals (Boylan et al. 2019). Furthermore, the effect of upbringing in a highly protective culture, such as that of Sri Lanka, with well-placed rules and norms for female behavior, may have negative effects on leadership skills. The authors have noted that the leadership roles in student societies and other extracurricular activities are taken by the male students in the faculty while the females carry out a supporting role. It will be very important to explore this gender difference in Sri Lanka and also in countries with similar cultures and upbringing and address this early in the curriculum since both males and females are expected to take leadership roles in the community.

A positive relationship between the leadership skills and the performance at the entry examination to medical school was observed. Acquisition of leadership skills prior to entering medical school could have occurred via various group activities done at the school level. It is also likely that good performance at this barrier examination requires some attributes of leadership skills measured via the ALQ. However, further studies are required to assess the effect of school level academic achievements on medical leadership.

Extracurricular activities seemed to have a positive effect on leadership skills. They help one to improve self-esteem, self-confidence, and to establish collaboration, interpersonal skills and the ability to work as a team member, which are integral components of leadership (Eccles et al. 2003; Zekan et al. 2012; Sari et al. 2014; Van Linden \& Fertman 1998). Thus, it's important to promote extracurricular activities from very early in life, especially at school level.

Despite the absence of formal leadership training in the curriculum, the results indicated that the students have developed leadership skills through the four years of study at the 
faculty. Exposure to actual clinical set up early in the career, good leadership role models and opportunities to work in groups could have contributed to this. However, the above contributors are yet to be confirmed through evidence-based research.

According to the current findings, majority had good knowledge on medical leadership while their leadership skill scores were not as high. However, the knowledge on leadership was significantly higher in the group of students with good leadership skills (i.e. ALQ score >3) compared to the counterpart, denoting the importance of developing their knowledge on leadership during the curriculum. It is also important to provide opportunities to translate knowledge into practice during their clinical training. (Kang \& Jin 2015; Goldstein et al. 2009).

Majority indicated that they would benefit from a formalized leadership training programme. According to a survey published by the University of Colorado, more than $80 \%$ of the participating institutions had no formalized training component on leadership in their curriculum and the rest of the institutions had optional modules (Alyssa 2017). Many studies have demonstrated the need of incorporation of leadership training in medical curriculum (True et al. 2020; Gulati et al. 2021; Till et al. 2020; Alyssa 2017) and various productive methods to develop leadership training such as simulation, interactive workshops, action learning, community and actual clinical set-upbased approaches have been introduced (Geerts et al. 2020; Saravo et al. 2017; Kiesewetter et al. 2013; Warren \& Carnall 2011; Goldstein et al. 2009). However, some researchers suggest that leadership training cannot be formalized and is mainly an experiential and personalized learning (Beer et al. 2016).

Current study demonstrated that with increasing age, changing in seniority and professional roles, participants had higher scores than their leaders. This may be so as with advancing age, workload and seniority student leaders may lose the motivation and skills to lead. Thus, Lack of leadership skills in the "leaders" could have contributed to the lower rating, highlighting the importance of providing continuous training in leadership skills (Blumenthal et al. 2012; Al-Moamary et al. 2016; Varkey et al. 2009).

Inability to assess the individual progress of the students as personal identification details were not obtained to ensure privacy could be stated as a limitation of this study.

\section{Conclusions}

It's prudent to integrate leadership training in undergraduate and postgraduate curricular of medical training while focusing on improving leadership skills in females as well. Further studies on how the medical leaders are perceived by the colleagues, students, healthcare teams and patients and what the stakeholders expect from the medical leaders would provide more insight into the issue at hand and would provide guidelines to plan training programs for medical leaders.

\section{Acknowledgement}

Research writing support programme (RWSP) of the Research Promotion and Facilitation Centre (RPFC) of the Faculty of Medicine, Colombo, mentored by the expertise from AuthroAID, UK, for the encouragement given to publish the finding and support given in language editing of the article.

\section{References}

Abbas MR, Quince TA, Wood DF, Benson JA: (2011) Attitudes of medical students to medical leadership and management: a systematic review to inform curriculum development. $B M C$ Medical education, 11, 1, p. 93.

Al-Moamary MS, Al-Kadri HM, Tamim HM: Authentic leadership in a health sciences university. Medical Teacher 2016, 38(sup1):S19-S25.

Alyssa B, Leadership in Medical Education.(cited 2017 Sep 13). Available at www.aamc.org/download/385246/data/leadershi pinmedicaleducation.pdf Accessed 13 Sep 2017.

Baker GR, Denis J-L: Medical leadership in health care systems: from professional authority to organizational leadership. Public Money \& Management 2011, 31, 5, pp. :355-362. 
Beer M, Finnstrom M, Schrader D: Why Leadership Tranning Fails-and What to Do About It. Harvard Business Review 2016, 94, 10, pp. 50-57.

Blumenthal DM, Bernard K, Bohnen J, Bohmer R: Addressing the leadership gap in medicine:residents' need for systematic leadership development training. Academic Medicine 2012, 87, 4, pp. 513-522.

Boylan, J., Dacre, J. and Gordon, H., 2019. Addressing women's under-representation in medical leadership. The Lancet, 393(10171), p.e14.

Eagly $\mathrm{AH}$, Johannesen-Schmidt MC, Van Engen ML: Transformational, transactional, and laissez-faire leadership styles: a meta-analysis comparing women and men. Psychol Bull. 2003; 129, 4, pp. 569-591.

Eccles JS, Barber BL, Stone M, Hunt J: Extracurricular activities and adolescent development. Journal of social issues 2003, 59, 4, pp. 865-889.

Geerts, J.M., Goodall, A.H. and Agius, S., 2020. Evidence-based leadership development for physicians: a systematic literature review. Social Science \& Medicine, 246, p.112709.

Goldstein AO, Calleson D, Bearman R, Steiner BD, Frasier PY, Slatt L: Teaching advanced leadership skills in community service (ALSCS) to medical students. Academic Medicine 2009, 84, 6, pp. 754-764.

Gulati, K., Madhukar, V., Verma, V., Singh, A.R., Gupta, S.K. and Sarkar, C., 2019. Medical leadership competencies: a comparative study of physicians in public and private sector hospitals in India. The International journal of health planning and management, 34, 1, pp.e947-e963.

Gulati, K., Sarkar, C., Verma, V., Singh, A.R. and Gupta, S.K., 2021. Assessment of medical leadership competencies and development needs: first comprehensive study from India. International Journal of Healthcare Management, 14, 2, pp.363-374.

Kang SK, Jin S: What are the Best Practices to Assess Leadership Effectiveness 2015. https://digitalcommons.ilr.cornell.edu/cgi/student ; accessed on 15th September 2017

Kiesewetter J, Schmidt-Huber M, Netzel J, Krohn AC, Angstwurm M, Fischer MR: Training of leadership skills in medical education. GMS Z Med Ausbild. 2013 Nov 15;30, 4, Doc49. Review.

Leadership and management for all doctors (2012) (Internet). GMC. https://www.gmc-uk.org/ethicalguidance/ethical-guidance-for-doctors/leader ship-and-management-for-all-doctors.Accessed 13 Sep 2017.
NHS Institute for Innovation and Improvement, 2010.The Medical Leadership Competency Framework; Enhancing Engagement in Medical Leadership. https://www.leadershipacademy.nh s.uk/wp-content/uploads/2012/11/NHSLeader ship-Leadership-Framework-Medical-Leader ship -Competency-Framework-3rd-ed.pdf

Saravo B, Netzel J, Kiesewetter J:The need for strong clinical leaders - Transformational and transactional leadership as a framework for resident leadership training. PLoS One. 2017 Aug 25;12, 8, e0183019.

Sarı I, Soyer F, Gülle M: The Role of Perceived Leadership Behaviors as Predictor of Assertiveness Levels in Individual Sport Athletes. Procedia - Social and Behavioral Sciences 2014, 152, pp. 446-450.

Spurgeon P, Barwell F, Mazelan P: Developing a medical engagement scale (MES). International Journal of Clinical Leadership 2008, 16,4.

Steinert $\quad \mathrm{Y}$, Naismith L, Mann K: Faculty development initiatives designed to promote leadership in medical education. A BEME systematic review: BEME Guide No. 19. Medical Teacher, 2012, 34, 6, pp. 483-503.

Till, A., McKimm, J. \& Swanwick, T., 2020. The importance of leadership development in medical curricula: a UK perspective (stars are aligning). Journal of healthcare leadership, 12, p.19.

True, M.W., Folaron, I., Colburn, J.A., Wardian, J.L., Hawley-Molloy, J.S. and Hartzell, J.D., 2020. Leadership training in graduate medical education: time for a requirement?. Military medicine, 185,1-2, pp.e11-e16.

Van Linden JA, Fertman Cl: Youth leadership: A guide to understanding leadership development in adolescents (!st Ed) Jossey-Bass; USA, 1998.

Varkey P, Peloquin J, Reed D, Lindor K, Harris I: Leadership curriculum in undergraduate medical education: a study of student and faculty perspectives. Med Teach. 2009 Mar;31, 3, pp 244-250. doi: 10.1080/01421590802144278

Walumbwa FO, Avolio BJ, Gardner WL, Wernsing TS, Peterson SJ: Authentic leadership: Development and validation of a theory-based measure. Journal of management 2008, 34, 1, pp. 89-126.

Warren OJ, Carnall R: Medical leadership: why it's important, what is required, and how we develop it. Postgraduate medical journal 2011, 87(1023), pp. 27-32.

Zekan SB, Peronja I, Russo A: Linking theory with practice: Students perceptions of leaders and leadership characteristics. Procedia - Social and Behavioral Sciences 2012, 41:237-242. 